\title{
La règle des trois unités dans la Place Royale de Pierre Corneille
}

\section{Dr. Adli Abdel Raouf ${ }^{(*)}$}

Où, quand, comment l'action de la pièce se déroule-t-elle? La règle des trois unités que les théoriciens du théâtre, reprenant les idées d'Aristote, commencent à établir vers 1930, et qui sera résumée plus tard, Boileau dans ces vers célèbres de L'Art Poétique:

Nous voulons qu'avec art l'action se ménage,

Qu'en un lieu, qu'en un jour, un seul fait accompli

Tienne jusqu'à la fin le théâtre rempli. ${ }^{1}$

Constitue, à l'époque classique, une réponse à ces questions. Nous allons voir si, lorsqu'il écrit La place Royale, Corneille suit cette règle dont le respect constitue une contrainte dramaturgique.

\section{A- Le lieu et le décor.}

"La scène est à la Place Royale" peut-on lire à la suite de la liste des acteurs. La Place Royale est à l'époque où la pièce fut créée un haut lieu parisien, situé au cœur du plus beau quartier de la ville: Marais. Corneille, faisant preuve de réalisme, place ses personnages dans un décor contemporain, familier du public. Il réalise un tableau de mœurs. Il fait allusion à la vie mondaine que l'on mène dans le quartier en

(*) Maître de Conférences

1 - Boileau (Nicolas): Art Poétique. Paris, Garnier, 1961, P. 172 
mentionnant le bal donné par Doraste chez Angélique pendant toute la durée du quatrième acte. Corneille mise aussi sur l'intérêt suscité chez le public par une pièce qui se déroule dans un cadre connu. Dans l'Examen de La Galerie du Palais, Corneille écrit: "J'ai pris ce titre de La Galerie Du Palais parce que la promesse de ce spectacle extraordinaire et agréable pour sa naïveté ${ }^{1}$ devait exciter vraisemblablement la curiosité des auditeurs" ${ }^{2}$ Sans doute s'est-il livré au même calcul en ce qui concerne le choix du titre de La Place Royale.

La Place Royale est un décor qui justifie la catégorie sociale à laquelle appartiennent les personnages, partant leurs comportement, leur langage, les acteurs dialoguent le plus souvent sur la place publique, et non chez eux, ce qui parait assez peu vraisemblable. Le respect de l'unité de lieu oblige à cette convention. Une jeune fille de haute noblesse ne pourrait s'entretenir dehors avec un jeune homme, une jeune fille de petite noblesse peut le faire sans choquer les convenances. Corneille établit cette distinction dans l'Examen de La Galerie du Palais: "Célidée et Hippolyte ... ne sont pas d'une condition trop élevée pour souffrir que leurs amants les entretiennent à leur porte. Il est vrai que ce qu'elles y disent serait mieux dit dans une chambre ou dans une salle..." ${ }^{3}$. Ce sont donc, selon l'attente du public, des jeunes gens aisés, oisifs, vêtus à la dernière mode qui évoluent dans le décor résidentiel de $\underline{\mathrm{La}}$

\footnotetext{
${ }^{1}$ Naïveté: infidélité de sa reproduction du réel.

2 - Corneille (Pierre): Théâtre complet. L'Examen de La Galerie du Palais, P. 329

3 -Ibid. P. 330
} 
Place Royale. L'élément visuel formé par de beaux costumes agréables n'est pas à négliger dans le succès d'une pièce. Le miroir pendu à la ceinture d'Angélique (Acte II, Scène 2) est un accessoire à la mode.

Le décor justifie la vraisemblance de l'action: les alentours de La Place Royale sont peu sûrs, la nuit venue, car infestés de bandes de brigands; Alidor invoque cette raison pour refuser d'enlever Angélique, seul, sans l'aide de ses gens:

Mon carrosse est parti, mes gens ont fait retraite;

A Paris, et de nuit, une telle beauté

Suivent un homme seul est mal en sûreté,

Doraste, ou par malheur quelque pire surprise

De ces coureurs de nuit me ferait lâcher prise. (Acte IV, scène 7)

De jour, la Place Royale est un endroit très fréquenté. C'est pourquoi Lycante se montre certain de retrouver Alidor, un jour ou l"autre, parmi les flâneurs:

Nous le rencontrerons, n'en soyez point en peine,

Où que soit sa retraite, il n'est pas toujours nuit Et ce qu'un jour nous cache un autre le produit. (Acte V, Scène 4)

L'action, en réalité exige deux décors: la Place Royale et le cabinet d'Angélique. La majeure partie de la pièce se déroule sur la place publique, mais la scène 5 de l'acte III se joue dans le cabinet d'Angélique, ainsi que le précise l'indication scénique placée en tête de la scène: "Angélique dans son cabinet". La scène suivante se déroule dans le même décor, précision qui est apportée par l'indication scénique: " Angélique voyant Alidor entrer en son cabinet". Dans l'Examen de la pièce; Corneille justifie l'emploi de deux décor: "Malgré 
cet abus introduit par la nécessité et légitimité par l'usage de faire dire dans la rue à nos amante de comédie ce que vraisemblablement elles diraient dans leurs chambre, je n'ai osé y placer Angélique durant la réflexion douloureuse qu'elle fait sur la promptitude et l'imprudence de ses ressentiments qui la font consentir à épouser l'objet de sa haine. J'ai mieux aimé rompre la liaison des scènes et l'unité de lieu qui se trouve assez exacte en ce poème, à cela près, afin de la faire soupirer dans son cabinet avec plus de bienséance pour elle et plus de sûreté pour l'entretien d'Alidor". 1 Corneille sacrifie donc l'unité de lieu à la bienséance. A la raison morale qu'il indique: sauvegarder la pudeur de sentiments de l'héroïne, Corneille ajoute une raison dramaturgique qui permet de sauvegarder la vraisemblance de l'action. L'entretien d'Angélique et Alidor doit avoir un caractère privé car "Philis qui le (Alidor) voit sortir de chez elle (Angélique) en aurait trop vu si elle les avait aperçus tous deux sur le théâtre; et au lieu...du soupçon de quelque intelligence renouée entre eux, qui la porte à l'observer durant le bal, elle aurait eu sujet d'en prendre une entière certitude, d'y donner un ordre qui eût rompu tout le nouveau dessein d'Alidor et l'intrique de la pièce". 2

Dans quel décor La Place Royale fut-elle jouée? On ne trouve pas trace du montage du décor de la pièce de Corneille, cependant, on peut imaginer la façon dont il se présentait, en lisant ce que Mahelot avait envisagé pour La Place Royale de Claveret, représentée à l'Hôtel de Bourgogne: "La feinteur doit

1. Corneille ( Pierre): La Place Royale. Examen. P. 114

2 - Ibid. P.P. 114-114 
faire paraître sur le théâtre la Place Royale ou l'imiter à peu près, et faire paraître un pavillon au milieu du théâtre, où sont les armes du roi et sous le pavillon, au travers de l'arche, faire paraître les minimes. A un côté de la place, une fenêtre où paraît quelqu'une."1 Pour la pièce de Corneille, on peut imaginer, à la place du pavillon, deux maisons d'angle, celle d'Angélique et celle de Philis, ou plus simplement encore, deux demeures placées côte-à-côte. Le décor n'est pas d'une importance primordiale. Il faut seulement que Cléandre et ses gens puissent se dissimuler, en attendant l'arrivée d'Angélique, sous les arcades qui supportent les habitations.

\section{Alidor}

Attends là de pied coy que je t'en avertisse.

(Il dit ce vers à Cléandre, et l'ayant fait

Retirer avec sa troupe il continue seul:) (Acte V, Scène 1)

L'indication scénique précise que la place, avec ses piliers et ses arcades offre une cachette providentielle. Dans la scène suivante, le texte reprend cette précision:

Alidor.

Qui te fait avancer?

Cléandre.

Je me lasse d'attendre.

Alidor

Laisse-moi, cher ami, le soin de t'avertir

En quel temps de ce coin il te faudra sortir. (Acte IV, Scène 2)

1 - H. C. Lancaster: Le mémoire de Mahelot, Laurent et autres décorateurs de l'Hôtel de Bourgogne. P. 95 
$\mathrm{Au} \mathrm{XVII}^{\mathrm{e}}$ siècle, les décors, très simples, se présentaient sous formes d'une ou plusieurs toiles peintes sommairement. $\mathrm{Si}$ un changement de décor devait intervenir, on tirait, au moment voulu, la première toile, faisant ainsi apparaître le décor suivant, placé derrière. C'est sans doute de cette façon que l'on a procédé pour effectuer le changement de décor nécessaire à la scène 5 du troisième acte, où, de la Place Royale, l'action se déplace dans le cabinet d'Angélique. On peut rapprocher ce décor de celui qui intervient dans Les Galanteries du Duc d'Ossonne de Mairet, à la scène 2 du deuxième acte: le duc qui attend devant la demeure d'une jeune fille qu'il courtise, en voit sortir un homme par une échelle de soi. Il monte par cette même échelle, et entre dans le cabinet de la jeune fille. Pour ce changement de décor, Mairet donne cette indication scénique: "comme il est entré la toile se tire, qui représente une façade de maison, et le dedans du cabinet parât". ${ }^{1}$ Le même principe est sans doute utilisé pour passer du décor de la Place Royale à celui du cabinet d'Angélique : Alidor entre chez la jeune fille, on tire la toile et cabinet se substitue à la place publique.

Le public du XVII ${ }^{\mathrm{e}}$ siècle se contentait d'un décor rudimentaire: son imagination pourvoyait à étouffer les quelques éléments présentés sur la scène.

\section{B-Le temps.}

Dans le discours des trois unités, Corneille écrit: "Le poème dramatique est...un portrait des actions des hommes. La représentation dure deux heures et ressemblerait parfaitement,

1 Jean Mairet: Les Galanteries du Duc d'Ossonne, Vice-Roy de Naples, Paris, Nizet, 1972, P. 21 
si l'action qu'elle représente n'en demandait pas davantage pour sa réalité...Si nous ne pouvons la renfermer dans ces deux heures, prenons-en quatre, six, dix, mais ne passons pas de beaucoup les vingt-quatre heures de peur de tomber dans le dérèglement..."1. Le respect de l'unité de temps est une contrainte dramaturgique qui agit sur la conception même de l'action: il faut que les divers mouvements de celle-ci puissent se dérouler avec vraisemblance dans le temps accordé par la règle. L'unité de temps agit également sur le choix du lieu de l'action: en vingt-quatre heures les personnages ne peuvent se déplacer sur les grandes distances.

De quelques indications données par le texte et la lecture de l'Examen de La Place Royale, nous pouvons conclure que du premier au dernier acte vingt-quatre se sont écoulées. En effet, dans l'Examen, Corneille donne les raisons pour lesquelles il n'a respecté ni l'unité de temps, ni l'unité d'action. Il ne mentionne pas l'unité de temps. De cette omission il aisé de tirer la conclusion suivante: Corneille a suivi la règle de l'unité de temps, la plus facile des trois, celle qui s'adapte sans trop de peine aux exigences de l'intrique. Nous pouvons supposer que le premier se déroule le matin. Pendant l'entracte Alidor élabore la ruse qui va lui permettre de pousser Angélique à la rupture. L'acte suivant se situe en début d'aprèsmidi. A la scène 5, Philis, voulant profiter du désarroi d'Angélique pour lui faire épouser Doraste, déclare: "Il faut donc se hâter, qu'elle (Angélique) ne refroidisse". (Acte II,

1 - P. Corneille: Théâtre complet, Discours des trois unités. P. 75 
Scène 5), ceci est une première indication de la précipitation de l'action. Cette précipitation se trouve confirmée un peu plus loin, lorsque Philis conseille à son frère hésitant: "Va, ne t'amuse point à savoir le pourquoi," (Acte II, Scène 5). Le temps presse, et Philis estime que des explications sur le changement d'humeur d'Angélique à l'égard d'Alidor retarderaient inutilement Doraste. Entre la scène $7 \mathrm{du}$ deuxième acte, dans laquelle Philis dit à Lisis: "Je te donne congé d'une heure si tu veux," et la scène 8 de l'acte suivant qui voit le retour de Lisis: "L'heure de mon congé n'est qu'à peine expirée, " une heure s'est donc écoulé. Il faut noter ici une tentative de rapprocher le temps réel du temps théâtral. Cette heure a été bien rempli puisqu'elle a vu Doraste obtenir la main d'Angélique: "Je possède Angélique" (III, 2) annonce-t-il triomphalement; puis Angélique regretter la "prompte vengeance" qui l'a conduite à accepter d'épouser Doraste; puis l'élaboration d'un nouveau stratagème par Alidor, et enfin la réconciliation d'Angélique et d'Alidor. Les événements se succèdent à une cadence très vive.

En tête du quatrième acte, une indication scénique précise qu'il "est dans la nuit". Dans la première scène, Alidor se félicite qu'"ebfin la nuit s'avance", car il doit retrouver Angélique "à minuit", ainsi que cela a été convenu plus tôt (III, 6). Cléandre, qui s'impatiente , car "minuit vient de sonner", nous donne dans la scène 2 , l'heure exacte.

Pendant l'entr'acte, la nuit se termine, Cléandre succombe au charme de Philis, enlevée par erreur. 
Le dernier acte se déroule le matin. Peu de Temps s'écoulé entre l'enlèvement de Philis et sa libération comme le prouve cette réplique de Lycante: "Mais Dieux! Voilà Philis qu'il (Cléandre ) a déjà rendu"( $\mathrm{V}, 4)$. La rapidité de l'action se trouve confirmée par la réplique d'Angélique s'adressant à son amie: "Ah, ma sœur,/ comme as-tu pu si tôt tromper ton ravisseur?"(V, 7).

Aussi, dans La Place Royale, l'action se déroule en vingtquatre heures: le premier acte commence le matin, le dernier acte se termine le matin suivant.

\section{C-L'unité d'action.}

L'unité d'action est l'unité qui a le plus d'importance dans la conception d'une pièce. Dans le Discours des trois unités, corneille écrit que " l'unité d'action consiste, dans la comédie, en l'unité d'intrique ou d'obstacle aux desseins des principaux acteurs". ${ }^{1}$ Il semble que cette unité ne soit pas respectée dans La Place Royale, car dans son Examen, nous pouvons lire: "Alidor (...) veut faire en sorte d'Angélique sa maîtresse se donne à son ami Cléandre (.. ) Ce dessein avorte et la donne à Doraste contre son intention et cela l'oblige à en faire un nouveau pour la porter à un enlèvement. Ces deux desseins, formés ainsi l'un après l'autre font deux actions et donnent deux âmes au poème.."2. L'action principale est double: Alidor veut contraindre angélique à rompre et il veut qu'elle épouse Cléandre. Cette duplicité de l'action permet d'ailleurs à Alidor d'affirmer sa valeur en triomphant d'obstacles successifs. Les

\footnotetext{
1 -Op.cit. P; 65

2 - Op. cit. P. 113
} 
obstacles ne naissent pas seulement de l'action principale, mais aussi de l'action secondaire: Philis agit pour favoriser l'amour que son frère porte à Angélique et réaliser le mariage souhaité par ce dernier. Elle s'oppose ainsi aux désirs d'Alidor. Corneille admet "plusieurs intriques ou obstacles (...) pourvu que de l'un on tombe nécessairement dans l'autre". 1

Jacques Scherer écrit que "l'action d'une pièce est unifiée lorsque l'intrique principale est dans un rapport tel avec les intriques accessoires que l'on puisse constater à la fois: $1^{0}$ qu'on ne peut supprimer aucune des intriques accessoires sans rendre partiellement inexplicable l'intrique principale; $2^{0}$ que toutes les intriques accessoires prennent naissance de le début de la pièce et se poursuivent jusqu'au dénouement; $3^{0}$ que le développement de l'intrique principale aussi bien que des intriques accessoires dépend exclusivement des données de l'exposition, sans introduction tardive d'événements dus hasard pur; $4^{0}$ que chaque intrique accessoire exerce une influence sur le déroulement de l'intrique principale". ${ }^{2}$

L'action de La Place Royale correspond bien à cette définition:

1- La suppression de l'intrique accessoire aboutirait à ce qu'Angélique, dépitée, accepte pour époux dès le second acte, alors que c'est Doraste, poussé par Philis, qu'elle accepte. Alidor n'aurait donc pas besoin de recourir à un second stratagème. Les doutes qui l'assailliront quant à savoir s'il garde Angélique ou la donne à Cléandre ne

1 -P; Corneille: Théâtre complet. Discours des trois unités. P. 65

2 - J. Scherer: La dramaturgie classique En France. P.P. 103-104 
naîtraient pas. Philis, surveillant Angélique, ne serrait pas enlevée par erreur, éveillant ainsi l'amour de Cléandre.

2- L'intrique accessoire prend naissance dès le premier acte et se poursuit jusqu'au dénouement dans lequel Doraste rend sa parole à Angélique.

3- Le développement de l'intrique principale et celui de l'intrigue secondaire dépendent de la situation initiale présentée dans l'exposition.

4- L'intrique secondaire influence le déroulement de l'intrique principale: par deux fois Philis fait échouer les plans d'Alidor.

L'unité de La Place royale consiste non pas dans une unité d'action, mais dans une unité d'intérêt, suscité par le héros principal qui, se heurtant à des obstacles, cherche à les surmonter.

Corneille, dans La Place royale, ne respecte pas la règle des trois unités, et semble s'en excuser dans l'Examen de 1660. Il juge alors sa pièce selon les critères de la doctrine classique. On peut juger de l'évolution de Corneille en comparant l'Examen avec ce qu'il écrivit dans l'Epitre de La suivante, en 1634: "J'aime à suivre les règles; mais loin de me rendre leur esclave, je les élargis et resserre selon qu'en a mon sujet,... Savoir les règles, et entendre les secret de les apprivoiser adroitement avec nôtre théâtre, ce sont deux sciences bien différentes; ..."1

1 - P. Corneille, Théâtre complet. Epitre de La Suivante. P. 414 
Ainsi, dans ses premières pièces, Corneille ne suit pas les règles avec obéissance aveugle: il les adapte selon les besoins de son sujet.

\section{Bibliographie}

- Boileau (Nicolas): Art Poétique. Paris, Garnier, 1961, P. 172

- Conesa (Gabriel) : Pierre Corneille et la naissance du genre comique (1629-1636)

- $\quad$ éd. Sedes, 1989.

- Corneille (Pierre): La Place Royale, éd. Gallimard, collection Folio Théâtre, 2006.

- Corneille (Pierre): Théâtre complet, Bibliothèque de la Pléiade, Paris , Gallimard, 1980

- Dort (Bernard: Pierre Corneille : dramaturge, L'Arche, 1957.

- Octave (Nadal): Le Sentiment de l'amour dans le théâtre de Pierre Corneille, éd. Mercure de France, 1951.

- Jean (Mairet): Les Galanteries du Duc d'Ossonne, ViceRoy de Naples, Paris, Nizet, 1972

- Lancaster (H. C.: Le mémoire de Mahelot, Laurent et autres décorateurs de l'Hôtel de Bourgogne. Paris, S. D.

- Schérer (Jacques): La dramaturgie Classique en France, Paris, Nizet, 1954 